\title{
PERTURBATION OF THE EIGENVALUES OF A MEMBRANE WITH A CONCENTRATED MASS*
}

\author{
BY \\ C. LEAL AND J. SANCHEZ-HUBERT \\ Université de Paris, France
}

\begin{abstract}
Résumé. Nous étudions une membrane vibrante avec une distribution de densité dépendant d'un petit paramètre $\varepsilon$, qui converge, lorsque $\varepsilon \searrow 0$, vers une densité uniforme plus une masse ponctuelle à l'origine. Nous mettons en évidence l'existence de vibrations locales, au voisinage de l'origine, et globales de la membrane. L'étude asymptotique lorsque $\varepsilon \searrow 0$ est effectuée à l'aide de la méthode des développements asymptotiques raccordés.

Abstract. We study a vibrating membrane with a distribution of density depending on $\varepsilon$, which converges, as $\varepsilon \searrow 0$, to a uniform density, plus a point mass at the origin. We establish local vibrations at the vicinity of the origin and global vibrations of the membrane. The asymptotic study for $\varepsilon \searrow 0$ is performed using the method of matched asymptotic expansions.
\end{abstract}

1. Introduction. We consider vibrating systems containing a small region, of diameter $O(\varepsilon)$, including the origin, where the density is very much higher than elsewhere. Quite different cases arise depending on the space dimension $N$ and the order of magnitude of the ratio $\varepsilon^{-m}$ of densities. Many studies are devoted to this problem of concentrated masses: E. Sanchez-Palencia [1], E. Sanchez-Palencia and H. Tchatat [2], H. Tchatat [3], O. A. Oleinik [4].

In this paper we study the case $N=2$ (i.e., the vibrating membrane) with $m \geq 2$. Using the method of matched asymptotic expansions (see for instance [5] and [6]), we derive the structure of the eigenfunctions, which is not given by other methods. It appears that there are two kinds of eigenvibration:

The local vibrations for which the corresponding eigenfunctions are of order $O(1)$ only in a region near the concentrated mass (i.e., for $|x|=O(\varepsilon)$ ) while elsewhere (i.e., for $|x|=O(1), x \neq 0)$ they are very small, of order $O(1 / \log \varepsilon)$. The corresponding eigenvalues are small, of order $\varepsilon^{m-2}$ for $m>2$, and of order $O(1)$ for $m=2$;

The global vibrations for which the corresponding eigenfunctions are of order $O(1)$ for $x=O(1), x \neq 0$, and become very small, of $\operatorname{order} O(1 / \log \varepsilon)$, in the vicinity ${ }^{*}$ Received November 24, 1987.

(C)1989 Brown University 
of the inclusion (i.e., for $|x|=O(\varepsilon)$ ). It may be said that these vibrations leave the concentrated mass almost at rest. The corresponding eigenvalues are $O(1)$.

We establish in Section 5 the existence of eigenvalues of order $\varepsilon^{m-2}$ (corresponding to the local vibration) in the case $m>2$.

2. Statement of the problem and first considerations about local vibrations. Let $\Omega$ be a bounded open domain of $\mathbf{R}^{2}$ with coordinates $x_{1}, x_{2}$. Denoting by $\varepsilon$ the small positive parameter, let $D$ be a bounded connected domain, with boundary $\Gamma$, of the auxiliary space $\mathbf{R}^{2}$ with coordinates $y_{1}, y_{2}$. In the $x_{1}, x_{2}$ space, we then consider the domain $\varepsilon D$, homothetic of $D$ with ratio $\varepsilon$. We suppose that both $\Omega$ and $D$ contain the origin as shown in Figure 1.
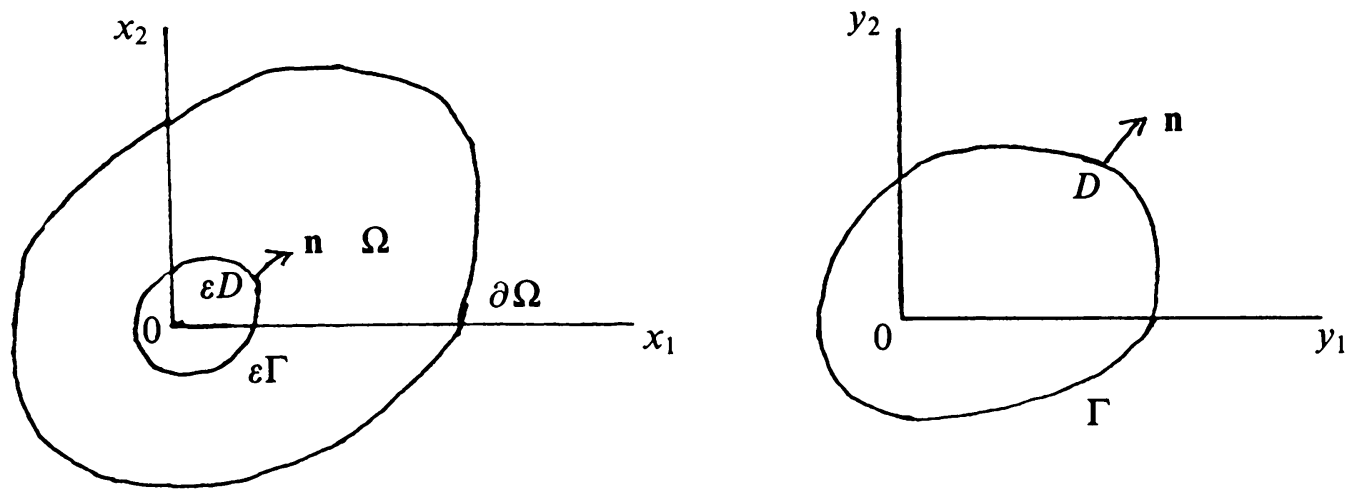

Fig. 1

We consider in $\Omega$ the eigenvalue problem:

$$
\begin{aligned}
-\Delta_{x} u^{\varepsilon} & =\lambda^{\varepsilon} \rho^{\varepsilon}(x) u^{\varepsilon} & & \text { in } \Omega, \\
u^{\varepsilon} & =0 & & \text { on } \partial \Omega,
\end{aligned}
$$

where

$$
\rho^{\varepsilon}(x)= \begin{cases}\varepsilon^{-m} & \text { for } x \in \varepsilon D, \\ 1 & \text { for } x \in \Omega \backslash \varepsilon \bar{D},\end{cases}
$$

for some $m>2$.

We note that the local concentrated mass is asymptotically very large with respect to the total mass of the membrane. 
It will prove useful to write down the problem separately in each one of two regions:

$$
\begin{aligned}
-\Delta_{x} u^{\varepsilon} & =\lambda^{\varepsilon} \varepsilon^{-m} u^{\varepsilon} & & \text { in } \varepsilon D, \\
-\Delta_{x} u^{\varepsilon} & =\lambda^{\varepsilon} u^{\varepsilon} & & \text { in } \Omega \backslash \varepsilon \bar{D}, \\
\llbracket u^{\varepsilon} \rrbracket & =0, \quad \llbracket \frac{\partial u^{\varepsilon}}{\partial n} \rrbracket=0 & & \text { on } \varepsilon \Gamma, \\
u^{\varepsilon} & =0 & & \text { on } \partial \Omega,
\end{aligned}
$$

where the transmission conditions (2.6) are associated with the Laplacian and the brackets denote the jump of the enclosed quantities.

Applying in (2.4)-(2.7) the dilatation $y=x \mid \varepsilon$ and defining the new spectral parameter

$$
\mu^{\varepsilon}=\varepsilon^{2-m} \lambda^{\varepsilon}
$$

we obtain:

$$
\begin{aligned}
-\Delta_{y} u^{\varepsilon} & =\mu^{\varepsilon} u^{\varepsilon} & & \text { in } D, \\
-\Delta_{y} u^{\varepsilon} & =\mu^{\varepsilon} \varepsilon^{n} u^{\varepsilon} & & \text { in } \varepsilon^{-1} \Omega \backslash \bar{D}, \\
\llbracket u^{\varepsilon} \rrbracket & =0, \quad \llbracket \frac{\partial u^{\varepsilon}}{\partial n} \rrbracket=0 & & \text { on } \Gamma, \\
u^{\varepsilon} & =0 & & \text { on } \partial\left(\varepsilon^{-1} \Omega\right) .
\end{aligned}
$$

Now, if we formally pass to the limit $\varepsilon \searrow 0$, we obtain a limiting problem of the form:

$$
\begin{aligned}
-\Delta_{y} u & =\mu u & & \\
-\Delta_{y} u & =0 & & \text { in } D, \\
\llbracket u \rrbracket & =0, \quad \llbracket \frac{\partial u}{\partial n} \rrbracket=0 & & \text { on } \Gamma,
\end{aligned}
$$

to which we shall append the condition at infinity (2.18), in order to get a well-posed problem.

In writing this limiting system, we suppose that $\mu^{\varepsilon}$ tends to a limit of order $O(1)$; for the time being, we assume this, postponing the proof until later (Sec. 5).

We first solve our problem in $\mathbf{R}^{2} \backslash \bar{D}$. Let $\varphi$ be a given function in $H^{1 / 2}(\Gamma)$. It is well known [7] that the problem

$$
\begin{aligned}
-\Delta_{y} u=0 & \text { in } \mathbf{R}^{2} \backslash \bar{D}, \\
u=\varphi & \text { on } \Gamma
\end{aligned}
$$

is well-posed upon appending the additional condition,

$$
u \rightarrow c \text { for }|y| \rightarrow+\infty,
$$

where $c$ is some constant.

Let $u^{\varphi}$ be the solution of $(2.16)-(2.18)$. As in [5] Section IV.8, we define the operator

$$
T \in \mathscr{L}\left(H^{1 / 2}(\Gamma), H^{-1 / 2}(\Gamma)\right)
$$


by

$$
T \varphi=-\frac{\partial u^{\varphi}}{\partial n} .
$$

The eigenvalue problem (2.13)-(2.15) is equivalent to the eigenvalue problem:

Find $\lambda \in \mathbf{R}, u \in H^{1}(D), u \neq 0$ such that

$$
a(u, w)=\mu \int_{D} u w d y \quad \forall w \in H^{\prime}(D),
$$

where the bilinear form $a$ is defined by

$$
a(u, w)=\int_{D} \frac{\partial u}{\partial y_{i}} \frac{\partial w}{\partial y_{i}} d y-\left\langle\left. T u\right|_{\Gamma},\left.w\right|_{\Gamma}\right\rangle
$$

We emphasize that the solution of (2.13)-(2.15) and (2.18) is the solution of (2.21) extended by $u^{u / \Gamma}$ to $\mathbf{R}^{2} \backslash \bar{D}$.

The form $a$ is continuous on $H^{1}(D)$ and symmetric. Moreover, $a(u, u) \geq 0$, $\forall u \in H^{1}(D)$. Indeed,

$$
a(u, u)=\int_{D}|\nabla u|^{2} d x+\int_{\mathbf{R}^{2} \backslash D}\left|\nabla u^{u_{\mid \Gamma}}\right|^{2} d x .
$$

We note that $\mu=0$ is an eigenvalue and the corresponding eigenfunction is constant. We are in the standard situation in which the eigenvalues of the limiting problem are

$$
0=\mu_{1}^{0}<\mu_{2}^{0} \leq \cdots \rightarrow+\infty .
$$

This suggests that, for $\varepsilon$ nonzero and small, there exist eigenvalues $\mu_{i}^{\varepsilon}$ such that

$$
\mu_{i}^{\varepsilon}=\mu_{i}^{0}+o(1)
$$

and thus the corresponding eigenvalues $\lambda_{i}^{\varepsilon}$ are of order $\varepsilon^{m-2}$. The corresponding eigenfunctions are approximately those of $a(u, w)=\mu(u, w)$ for fixed $y$, extended in a convenient way to $|x|=O(1)$. We claim that they are local vibrations in the sense that the corresponding eigenfunctions are small outside of $|y|=O(1)$. We shall show this later. In addition, global vibrations must exist.

3. Study of the global vibrations. The eigenvibration of the membrane is affected by the presence of the concentrated mass. As shown in [5], Sec. VII.12, the eigenvalues and eigenfunctions converge, as $\varepsilon \searrow 0$, towards those of the problem without the concentrated mass. This convergence is very weak and ignores the behaviour of the eigenfunctions in a neighbourhood $|x|=O(\varepsilon)$ of the inclusion. We use the method of matched asymptotic expansions to describe this behaviour in a precise manner.

We search, for $\lambda^{\varepsilon}$ and $u^{\varepsilon}$, with asymptotic expansions of the form:

$$
\begin{aligned}
& \lambda^{\varepsilon}=\lambda^{0}+o(1), \\
& u^{\varepsilon}=u^{0}+o(1) .
\end{aligned}
$$

By substituting (3.1) and (3.2) into (2.4)-(2.7), we obtain:

$$
\left\{\begin{aligned}
-\Delta_{x} u^{0} & =\lambda^{0} u^{0} \quad \text { in } \Omega \backslash\{0\} \\
\left.u^{0}\right|_{\partial \Omega} & =0
\end{aligned}\right.
$$


In the spirit of the method of matched asymptotic expansions, we perform the dilatation $y=x \mid \varepsilon$ in order to study the solution in a vicinity of the origin. In this region we search, for $u^{\varepsilon}$, through an asymptotic expansion of the form

$$
u^{\varepsilon}=\eta(\varepsilon) v^{0}(y)+\cdots .
$$

By substituting (3.4) into (2.9)-(2.12), we obtain, on account of $\mu^{\varepsilon}=\varepsilon^{2-m} \lambda^{0}+\cdots$, for $m>2$,

$$
\begin{aligned}
v^{0}=0 & \text { in } D, \\
-\Delta_{y} v^{0}=0 & \text { in } \mathbf{R}^{2} \backslash D, \\
\llbracket v^{0} \rrbracket=0 & \text { on } \Gamma,
\end{aligned}
$$

inn. $\lim . u^{0}(x)=$ out. $\lim . \eta(\varepsilon) v^{0}(y)$.

We recall that inner (resp., outer) limit means limit as $\varepsilon \searrow 0$, for fixed $y$ (resp., fixed $x$ ).

The second transmission condition $\llbracket \frac{\partial v^{0}}{\partial n} \rrbracket=0$ on $\Gamma$ may be disregarded. Indeed, by considering (2.13), on account of $\mu^{\varepsilon}=\varepsilon^{2-m} \lambda^{0}+\cdots$, we see that the vibration in $D$ is of high frequency. Consequently, the wave length of the oscillations in $D$ is short and $\frac{\partial v^{0}}{\partial n}$ on $\Gamma$ does not make sense. This is analogous to the situation in the three-dimensional case ([5], Sec. VII.10).

Let us take, following the considerations at the beginning of this section, as solution of (3.3) the eigenvibration of $\Omega$ without singularity at the origin. After normalization, $u^{0}(x)$ is uniquely defined and

$$
\text { inn. } \lim . u^{0}(x)=\lim _{x \rightarrow 0} u^{0}(x)=u^{0}(0)
$$

is already known and in general it is different from zero. Consequently, the problem (3.5)-(3.8) has no solution without singularity as $|y| \rightarrow \infty$. We thus look for the weakest singular solution of

$$
\begin{aligned}
&-\Delta_{y} v^{0}=0 \quad \text { in } \mathbf{R}^{2} \backslash \bar{D}, \\
&\left.v^{0}\right|_{\Gamma}=0, \\
& \lim _{\varepsilon \backslash 0, \text { fixed } x} \eta(\varepsilon) v^{0}(y)=u^{0}(0),
\end{aligned}
$$

with known $u^{0}(0)$. We know [8] that the weakest singularity at infinity is logarithmic and, consequently, the solution has the following behaviour:

$$
v^{0}(y) \simeq-\frac{c}{2 \pi}(\log |y|+w(y))
$$

at infinity, where $w(y)$ is a nonsingular function as $|y| \rightarrow+\infty$. For $\varepsilon \backslash 0$, fixed $x$,

$$
\eta(\varepsilon) v^{0}(y) \sim-\frac{c}{2 \pi} \eta(\varepsilon) \log |y| \text { as }|y| \rightarrow \infty
$$

from which

$$
\lim _{\varepsilon \backslash 0, \text { fixed } x}\left[-\frac{c}{2 \pi} \eta(\varepsilon)(\log |x|-\log \varepsilon)\right]=u^{0}(0)
$$


and

$$
\begin{gathered}
c=2 \pi u^{0}(0), \quad \eta(\varepsilon)=\frac{1}{\log \varepsilon} \\
\eta(\varepsilon) v^{0}(y)=-\frac{u^{0}(0)}{\log \varepsilon}(\log |y|+w(y))
\end{gathered}
$$

where $w(y)$ is the solution of

$$
\left\{\begin{array}{c}
-\Delta_{y} w=0 \text { in } \mathbf{R}^{2} \backslash \bar{D} \\
w_{\mid \Gamma}=-\left.\log |y|\right|_{\Gamma} \\
w \rightarrow \text { const. for }|y| \rightarrow+\infty
\end{array}\right.
$$

This last problem is well-posed and, in particular, the constant at infinity is determined.

\section{Asymptotic study of the local vibration.}

The local vibration (i.e., the vibration of the inclusion itself) may only be studied after a dilatation of the domain $\varepsilon D$ that it occupies. Consequently, it is determined by the system (2.9)-(2.12). We shall see in Sec. 5 that the equivalent variational formulation of this problem is of the form

$$
a(\varepsilon, \mu, u, v)=\mu(u, v) \quad \forall v \in H^{1}(D) .
$$

We thus have a problem with implicit eigenvalues. We shall show that the eigenvalues $\mu^{\varepsilon}$ depend continuously on $\varepsilon$ and converge as $\varepsilon \searrow 0$ towards the eigenvalues $\mu_{i}(0)=\mu_{i}^{0}$ of the problem:

$$
\begin{aligned}
& -\Delta_{y} \tilde{v}^{0}=\mu^{0} \tilde{v}^{0} \quad \text { in } D, \\
& -\Delta_{y} \tilde{v}^{0}=0 \quad \text { in } \mathbf{R}^{2} \backslash \bar{D} \text {, } \\
& \llbracket \tilde{v}^{0} \rrbracket=0, \quad \llbracket \frac{\partial \tilde{v}_{0}}{\partial n} \rrbracket=0 \quad \text { on } \Gamma, \\
& \tilde{v}^{0} \rightarrow \tilde{c} \quad \text { for }|y| \rightarrow+\infty,
\end{aligned}
$$

as obtained by inserting asymptotic expansions of the form:

$$
\left\{\begin{array}{l}
u^{\varepsilon}=\tilde{v}^{0}+o(1) \\
\mu^{\varepsilon}=\mu^{0}+o(1)
\end{array}\right.
$$

for $u^{\varepsilon}$ and $\mu^{\varepsilon}$ in the vicinity of $\varepsilon D$.

We recognize here the limit problem considered in Sec. 2, the solution of which must be matched with the solution of the outer problem.

In the outer region, we look for an expansion of the form

$$
u^{\varepsilon}=\eta(\varepsilon) \tilde{u}^{0}(x)+\cdots
$$

which gives, by substituting into (2.4)-(2.7),

$$
\begin{gathered}
-\Delta_{x} \tilde{u}^{0}-\varepsilon^{m-2} \mu^{0} \tilde{u}^{0}=0 \quad \text { in } \Omega \backslash\{0\}, \\
\tilde{u}^{0}=0 \text { on } \partial \Omega, \\
\text { out. } \lim . \tilde{v}^{0}(y)=\text { inn. } \lim . \eta(\varepsilon) \tilde{u}^{0}(x) .
\end{gathered}
$$


For $m>2$ we thus have

$$
-\Delta_{x} \tilde{u}^{0}=0 \text { in } \Omega \backslash\{0\} .
$$

The only regular solution of this problem vanishes identically, so we take for $\tilde{u}^{0}(x)$ the weakest singular solution of the inner Dirichlet problem, i.e.,

$$
\tilde{u}^{0}(x)=-\frac{\tilde{A}}{2 \pi}[\log (|x|+f(x))],
$$

where $f(x)$ is regular at $x=0$. Then, from

$$
\begin{aligned}
\lim _{\varepsilon \backslash 0, \text { fixed } y} \eta(\varepsilon) \tilde{u}^{0}(\varepsilon y) & =-\frac{A}{2 \pi} \eta(\varepsilon)[\log |y|+\log \varepsilon+f(\varepsilon y)] \\
& =\lim _{|y| \rightarrow+\infty} \tilde{v}^{0}(y)=\tilde{c}
\end{aligned}
$$

we obtain

$$
\tilde{A}=-2 \pi \tilde{c}, \quad \eta(\varepsilon)=1 / \log \varepsilon,
$$

from which

$$
\eta(\varepsilon) \tilde{u}^{0}(x)=\frac{2 \pi \tilde{c}}{\log \varepsilon}[\log |x|+f(x)] .
$$

Here $f(x)$ is the solution of the problem

$$
\begin{gathered}
-\Delta_{x} f=0 \quad \text { in } \Omega, \\
f(x)_{\mid \partial \Omega}=-\log |x|_{\partial \Omega},
\end{gathered}
$$

which is well-posed.

\section{Rigorous study of the eigenvalues of the local vibration.}

We establish the existence of the eigenvalues $\lambda^{\varepsilon}$ of the form $\lambda^{\varepsilon}=\varepsilon^{m-2} \mu^{\varepsilon}$ and the convergence of $\mu_{i}^{\varepsilon}$ to the eigenvalues $\mu_{i}(0)$ of the limiting problem. A result in this direction was announced in [4]. As in [5], we write the eigenvalue problem (2.9)(2.12) by taking $\zeta \in \mathbf{C}$ as a spectral parameter:

$$
\begin{aligned}
& -\Delta_{y} u=\zeta u \quad \text { in } D \text {, } \\
& -\Delta_{y} u=\zeta \varepsilon^{m} u \quad \text { in } \varepsilon^{-1} \Omega \backslash D \text {, } \\
& \llbracket u \rrbracket=0, \quad \llbracket \frac{\partial u}{\partial n} \rrbracket=0 \text { on } \Gamma, \\
& u=0 \quad \text { on } \partial \varepsilon^{-1} \Omega .
\end{aligned}
$$

The limiting problem, obtained by letting $\varepsilon \searrow 0$, reads:

$$
\begin{aligned}
& -\Delta_{y} u=\zeta u \quad \text { in } D, \\
& -\Delta u=0 \quad \text { in } \mathbf{R}^{2} \backslash D \text {, } \\
& \llbracket u \rrbracket=0, \quad \llbracket \frac{\partial u}{\partial n} \rrbracket=0 \text { on } \Gamma,
\end{aligned}
$$

where condition (5.4) must be replaced by

$$
u \rightarrow c \text { for }|y| \rightarrow+\infty, \quad c=\text { const }
$$

for well-posedness in $\mathbf{R}^{2} \backslash D$.

We have 
TheOREM. The eigenvalues $\mu_{i}(\varepsilon)$ of the problem (5.1)-(5.4) with $m>2$ depend continuously on $\varepsilon$ and converge as $\varepsilon \searrow 0$ to the eigenvalues $\mu_{i}(0)$ of the limit problem (5.5)-(5.8).

Proof. Up to slight modifications, the proof is the same as that of Proposition VII.11.4 in [5]. The reader is referred to that work for details. We only present here an outline.

The limit problem (5.5)-(5.8) was transformed, in Sec. 2, into another problem on $D$. We make an analogous transformation for the problem (5.1)-(5.4). To this end, we solve

$$
\begin{aligned}
-\Delta_{y} u^{\ell \zeta} & =\zeta \varepsilon^{m} u^{\ell \zeta} & & \text { in } \varepsilon \Omega^{-1} \backslash \bar{D}, \\
u^{\ell \zeta} & =0 & & \text { on } \partial \varepsilon^{-1} \Omega, \\
u^{\ell \zeta} & =\varphi & & \text { on } \Gamma .
\end{aligned}
$$

This problem may be uniquely solved for $m$ and $\zeta \in \Delta$, where $\Delta$ is some bounded domain of $\mathbf{C}$. Indeed, $\zeta \varepsilon^{m}$ is not an eigenvalue of this problem because the Poincare constant is of the form $\varepsilon^{-1} C(\Omega)$.

Then, we may define $T(\varepsilon, \zeta)$ as in (2.19). It is an operator in $\mathscr{L}\left(H^{1 / 2}(\Gamma), H^{-1 / 2}(\Gamma)\right)$. We now define the form $a$ by:

$$
a(\varepsilon, \zeta ; u, v)=\int_{D} \frac{\partial u}{\partial y_{i}} \frac{\partial v}{\partial y_{i}} d y+\left\langle\left. T(\varepsilon, \zeta) u\right|_{\Gamma},\left.v\right|_{\Gamma}\right\rangle
$$

The eigenvalue problem (5.1) is equivalent to the implicit eigenvalue problem

$$
a(\varepsilon, \zeta ; u, v)=\zeta(u, v)_{L^{2}(D)} \quad \text { in } H^{1}(D) .
$$

In analogy to the limiting problem (2.20), the corresponding operator and form will be denoted by $T(0)$ and $a(0 ; u, v)$. The assertion of the theorem then follows from [5] (Proposition V.10.6 and Remark V.10.7). Indeed the hypotheses of this proposition are satisfied by virtue of the following lemma.

LemMA. If, as $j \rightarrow+\infty$,

$$
\begin{aligned}
& \varphi^{j} \rightarrow \varphi^{*} \quad \text { in } H^{1 / 2}(\Gamma) \text { weakly, } \\
& \varepsilon_{j} \rightarrow 0 \\
& \zeta_{j} \rightarrow \zeta^{*} \in \Delta
\end{aligned}
$$

then

$$
T\left(\varepsilon_{j}, \zeta_{j}\right) \varphi^{j} \rightarrow T(0) \varphi^{*} \quad \text { in } H^{-1 / 2}(\Gamma) \text { weakly. }
$$

Proof. We define $W$ as the completion of $\mathscr{D}\left(\varepsilon^{-1} \Omega \backslash \bar{D}\right)$ under the Dirichlet norm. By [9], in the two-dimensional case, $W$ contains functions which take nonzero value in a neighbourhood of infinity. Bearing this in mind, the proof is the same as in [5], Lemma VII.11.3. 
6. Study of the case $N=2, m=2$. In the case $m=2$, the concentrated mass is of the same order as the total mass of the membrane. This case exhibits features analogous to the previous case $m>2$ but the two sequences of eigenvalues $\lambda_{i}^{0}$ (resp., $\mu_{i}^{0}$ ) associated with the global (resp., local) vibration are of the same order $O(1)$. This entails some new difficulties. We only study the eigenvalues of each sequence which are simple and different from those of the other sequence.

The limiting problem in the $x$-variables reads

$$
\left\{\begin{aligned}
-\Delta_{x} u^{0} & =\lambda^{0} u^{0} \quad \text { in } \Omega \backslash\{0\} \\
\left.u^{0}\right|_{\partial \Omega} & =0
\end{aligned}\right.
$$

and in the $y$-variables reads

$$
\begin{aligned}
& -\Delta_{y} v^{0}=\mu^{0} v^{0} \quad \text { in } D, \\
& -\Delta_{y} v^{0}=0 \quad \text { in } \mathbf{R}^{2} \backslash \bar{D} \text {, } \\
& \llbracket v^{0} \rrbracket=0, \quad \llbracket \frac{\partial v^{0}}{\partial n} \rrbracket=0 \text { on } \Gamma, \\
& v^{0} \rightarrow c \quad \text { for }|y| \rightarrow+\infty .
\end{aligned}
$$

Let us study the local vibration. We saw in Sec. 2 that the eigenvalue problem (6.2) is a standard one with eigenvalues

$$
0=\mu_{1}^{0}<\mu_{2}^{0}<\cdots \rightarrow+\infty
$$

and associated eigenfunctions $v_{1}^{0}, v_{2}^{0}, \ldots$ After normalization, the corresponding constants $c_{i}$ are determined.

We search for the corresponding outer limit in the form $\eta(\varepsilon) u^{0}(x)$ satisfying

$$
\begin{aligned}
& -\Delta_{x} u^{0}=\mu^{0} u^{0} \quad \text { in } \Omega \backslash\{0\}, \\
& \left.u^{0}\right|_{\partial \Omega}=0, \\
& \eta(\varepsilon) u^{0}(x) \rightarrow c \quad \text { for } \varepsilon \searrow 0, \text { fixed } y .
\end{aligned}
$$

The singularity at the origin is logarithmic as in Sec. 4. Indeed, the fundamental solution is expressed by Bessel functions which have logarithmic singularity at the origin.

We look for $u^{0}(x)$ in the form:

$$
u^{0}(x)=-\frac{1}{2 \pi} \log |x|+f(x)
$$

where $f(x)$ is regular everywhere in $\Omega$.

From the matching condition (6.5) we obtain $\eta(\varepsilon)=-2 \pi c / \log \varepsilon$.

From (6.3) and (6.4) $f(x)$ is the solution of

$$
\left\{\begin{array}{l}
-\Delta_{x} f=\mu^{0}\left[-\frac{1}{2 \pi} \log |x|+f(x)\right] \text { in } \Omega, \\
\left.f\right|_{\partial \Omega}=\left.\frac{1}{2 \pi} \log |x|\right|_{\partial \Omega} .
\end{array}\right.
$$

This is a well-posed problem since $\mu^{0}$ is not an eigenvalue of $(6.1)$. 
Let us now consider the global vibration. We take as eigenvalues and eigenfunctions of (6.1) those of the eigenvibration of the membrane without inclusion. Then the eigenvalues $\lambda_{i}^{0}$ of this problem, which are assumed known, satisfy

$$
0 \neq \lambda_{1}^{0} \leq \lambda_{2}^{0} \leq \cdots \rightarrow+\infty \text {. }
$$

After normalization, the $u_{i}^{0}(x)$ are well-determined.

We search for the corresponding inner expansion of the form $\eta(\varepsilon) v^{0}(y)$ satisfying

$$
\begin{aligned}
& -\Delta_{y} v^{0}=\lambda^{0} v^{0} \quad \text { in } D \text {, } \\
& -\Delta_{y} v^{0}=0 \quad \text { in } \mathbf{R}^{2} \backslash \bar{D} \text {, } \\
& \llbracket v^{0} \rrbracket=0, \quad \llbracket \frac{\partial v^{0}}{\partial n} \rrbracket=0 \text { on } \Gamma, \\
& \lim _{\varepsilon \backslash 0, \text { fixed } x} \eta(\varepsilon) v^{0}(y)=u^{0}(0),
\end{aligned}
$$

where $\lambda^{0}$ and $u^{0}(0)$ are known.

We seek $v^{0}$ with the weakest singularity at infinity, of the form

$$
v^{0}(y)= \begin{cases}v(y) & \text { in } D \\ \log |y|+w(y) & \text { in } \mathbf{R}^{2} \backslash \bar{D}\end{cases}
$$

where $w(y) \rightarrow$ const. for $|y| \rightarrow+\infty$.

The system (6.3)-(6.6) then becomes

$$
\left\{\begin{array}{c}
-\Delta_{y} v=\lambda^{0} v \quad \text { in } D, \\
-\Delta_{y} w=0 \quad \text { in } \mathbf{R}^{2} \backslash \bar{D}, \\
\left.v\right|_{\Gamma}=\left.\log |y|\right|_{\Gamma}+\left.w\right|_{\Gamma},\left.\quad \frac{\partial v}{\partial n}\right|_{\Gamma}=\frac{\partial}{\partial n} \log |y|_{\Gamma}+\left.\frac{\partial w}{\partial n}\right|_{\Gamma}, \\
\lim _{\varepsilon \backslash 0, \text { fixed } x} \eta(\varepsilon) v^{0}(y)=u^{0}(0) .
\end{array}\right.
$$

Setting $\varphi=\left.v\right|_{\Gamma}$ we have

$$
\left\{\begin{aligned}
&-\Delta_{y} w=0 \text { in } \mathbf{R}^{2} \backslash D, \\
&\left.w\right|_{\Gamma}=\varphi-\left.\log |y|\right|_{\Gamma}, \\
& w \rightarrow c \text { (unknown constant) for }|y| \rightarrow \infty .
\end{aligned}\right.
$$

Denoting by $w$ the solution of this problem and computing $\frac{\partial}{\partial n}(w+\log |y|)$ on $\Gamma$, the problem in $D$ becomes

$$
\left\{\begin{array}{l}
\text { Find } v \in H^{1}(D) \text { satisfying } \\
a(v, \chi)-\lambda^{0}(v, \chi)=\left\langle T\left(\log |y|_{\Gamma}\right)+\left.\frac{\partial}{\partial n} \log |y|\right|_{\Gamma},\left.\chi\right|_{\Gamma}\right\rangle,
\end{array}\right.
$$

where $T$ was defined in Sec. 2 and where

$$
a(v, \chi) \equiv \int_{D} \nabla v \nabla \chi d x+\left\langle\left. T v\right|_{\Gamma},\left.\chi\right|_{\Gamma}\right\rangle
$$

Since $\lambda^{0}$ is not an eigenvalue of the corresponding homogeneous problem, the problem (6.11) has a unique solution $v$, and then $\left.v\right|_{\Gamma}$ is well-defined and so is $w$.

It only remains to perform the matching:

$$
\lim _{\varepsilon \backslash 0, \text { fixed } x} \eta(\varepsilon)(\log |y|+w(y))=u^{0}(0),
$$

which gives, as before, $\eta(\varepsilon)=-u^{0}(0) / \log \varepsilon$. 
Remark. On account of the definition of $T$ one may think that $T\left(\left.\log |y|\right|_{\Gamma}\right)+$ $\frac{\partial}{\partial n} \log \mid y \|_{\Gamma}$ vanishes. But this is false. Indeed, the solution of the outer problem tends to a constant at infinity. The solution with given $\log \mid y \|_{\Gamma}$ is not $\log |y|$ but a function the normal derivative of which is, by definition, $T\left(\log \mid y \|_{\Gamma}\right)$.

\section{REFERENCES}

[1] E. Sanchez-Palencia, Perturbation of eigenvalues in thermoelasticity and vibration of systems with concentrated masses, In Trends in applications of pure mathematics to mechanics, P. Ciarlet and $\mathrm{M}$. Roseau, editors, Springer, 1984

[2] E. Sanchez-Palencia and $\mathrm{H}$. Tchatat, Vibration de systèmes élastiques avec masses concentrées, Rend. Sem. Mat. Univ., Politecn. Torino, 42, 43-63 (1984)

[3] H. Tchatat, Thèse de 3ème cycle, University Pierre et Marie Curie, 1984

[4] O. A. Oleinik, Homogenization problems in elasticity. Spectra of singularly perturbed operators in Nonclassical continuum mechanics, Ed. R. J. Knops and A. A. Lacey, Cambridge Univ. Press, 80-95 (1987)

[5] J. Sanchez-Hubert and E. Sanchez-Palencia, Vibration and coupling of continuous systems. Asymptotic methods, Springer, Heidelberg (To be published 1988-89)

[6] J. D. Cole and J. Kevorkian, Perturbation methods in applied mathematics, Springer

[7] G. Goursat, Cours d'Analyse mathématique, Gauthier-Villars, Paris, Vol. 3 (1921)

[8] R. Dautray and J. L. Lions, Analyse mathématique et calcul numérique pour les sciences et les techniques, Tome 1, Masson, Paris

[9] O. A. Ladyzenskaia, Mathematical theory of viscous fluid flows, Gordon and Breach, New York, 1963 